Check for updates

Cite this: RSC Adv., 2019, 9, 27449

\title{
Characterization of antioxidant and antibacterial gelatin films incorporated with Ginkgo biloba extract
}

\begin{abstract}
Xiaona Hu, ${ }^{\text {ab }}$ Li Yuan, $^{\text {ab }}$ Lijun Han, $^{\text {ab }}$ Shujing Li ${ }^{*}{ }^{* a b}$ and Liya Song ${ }^{* b c}$
Ginkgo biloba extract (GBE) contains Ginkgo biloba flavonoids, which are phenolic compounds. These compounds can be introduced into films for their functional properties (such as their antioxidant and antibacterial property), allowing this film to be used as food packaging. Thus, the aim of this study was to introduce the GBE into a gelatin solution to prepare gelatin films and evaluate the influence of the natural extract addition on the physicochemical and antimicrobial properties. The gelatin films were successfully prepared by casting technique, and GBE was incorporated at concentrations of $0,0.5,1.5$, $2.5,3,4$, and $5 \mathrm{~g} / 100 \mathrm{~g}$ of gelatin. The mechanical properties, film solubility, moisture content, water vapor permeability, infrared spectroscopic characteristics, film microstructure, light barrier property, antioxidant property and antibacterial property of the films were investigated. The incorporation of gelatin films with GBE increased the UV-visible shielding performance of films. The antioxidant ability of the film was improved, which was supposed to be related to the active substances of the GBE. The GBE also exhibited potential antimicrobial activity against tested microorganisms. With the increase in the GBE concentration incorporated into the films, the antimicrobial activity of the gelatin film with GBE was also enhanced.
\end{abstract}

Received 26th July 2019

Accepted 11th August 2019

DOI: $10.1039 / c 9 r a 05788 a$

rsc.li/rsc-advances showed 20-fold increase in antioxidant activity and 6-fold increase in antibacterial activity as compared to those of the control film. Besides, the releasable caffeic acid can be continuously released from the films, and the lipid oxidation of the menhaden oil in water emulsion is significantly inhibited. ${ }^{5}$ Jouki et al. found that quince seed mucilage films containing thyme essential oil increased the percent elongation of films. Moreover, the additivated films presented good antioxidant activity and significant antibacterial activity. ${ }^{6}$

Ginkgo biloba is the oldest living relict plant with both ecological and medicinal value . ${ }^{7}$ Ginkgo biloba extract (GBE) is obtained by refluxing ginkgo leaves in ethanol. The main chemical components of ginkgo leaves can be divided into two types: flavonoids and terpene lactones. Flavonoids can be divided into three types: flavones, diflavones, and catechins, which are mainly in the form of glucosides. Terpenoid lactones include sesquiterpene lactones, diterpene lactones, and triterpene lactones. ${ }^{8}$ These chemical components show extensive pharmacological activity.

Previous studies have demonstrated that GBE is known for its antioxidant as well as strong antibacterial properties, ${ }^{9}$ but there is no report about its application in food packaging. The aim of this work is to use the GBE to prepare gelatin films and evaluate the effects of GBE incorporation on the antioxidant and antimicrobial properties of the gelatin films. Furthermore, the physicochemical properties (such as mechanical properties,

\footnotetext{
${ }^{a}$ Department of Chemistry, School of Science, Beijing Technology and Business University, Beijing 100048, PR China. E-mail: lishujing@th.btbu.edu.cn

${ }^{b} A$ Key Laboratory of Cosmetic, Beijing Technology and Business University, China National Light Industry, Beijing, 100048, PR China

${ }^{c}$ Beijing Key Lab of Plant Resources Research and Development, Beijing Technology and Business University, Beijing 100048, PR China
} 
surface structure, and UV shielding effect) of the films with the addition of GBE were also evaluated.

\section{Methods and materials}

\subsection{Materials}

Gelatin and glycerol were purchased from Sinopharm Group Co., Ltd. Ginkgo biloba extract (GBE) was bought from Nanjing Herb Source Bio-Tech Co., Ltd. 2,2-Diphenyl-1-picrylhydrazyl (DPPH) was supplied by TCI (Shanghai) Development Co., and Rose Bengal medium, nutrient broth and nutrition agar were supplied by AOBOXING Bio-Tech Co., Ltd. (Beijing, China).

\subsection{Preparation of gelatin film incorporated with GBE}

The GBE gelatin films were prepared by the method reported by Li et al. with some modifications. ${ }^{10}$ Briefly, gelatin was dissolved in distilled water to obtain a protein concentration of $4 \%(\mathrm{w} / \mathrm{v})$. The mixture was hydrated at room temperature for $60 \mathrm{~min}$, and then heated at $45^{\circ} \mathrm{C}$ until it dissolved completely. Based on the gelatin weight, $30 \%(\mathrm{w} / \mathrm{w})$ glycerol as a plasticizer was added to the film forming solution. In the meantime, concentrations of $0,0.5,1.5,2.5,3,4$, and $5 \%(\mathrm{w} / \mathrm{w}$, based on the gelatin content) GBE solubilized with ethanol were added to the gelatin solution. The film forming solutions were poured onto acrylic sheets $(10$ $\times 10 \mathrm{~cm}$ ). The solutions were dried in an oven (FED 115, Binder, Germany) at $25{ }^{\circ} \mathrm{C}$ for 3 days.

\subsection{Determination of film properties}

2.3.1. Mechanical properties. Tensile strength (TS) and elongation at break (EAB) of gelatin films were tested as described by Iwata $e t$ al. using an electromechanical universal testing machine (CMT 6104, MTS Systems Co., Ltd, China) equipped with a tensile load cell of $100 \mathrm{~N}$. The sample was cut into $25 \mathrm{~mm} \times 100 \mathrm{~mm}$ pieces. The cross-head speed was set at $10 \mathrm{~mm} \mathrm{~min}^{-1} .^{11}$

2.3.2. Moisture content and film solubility. The moisture content (MC) and film solubility (FS) were measured by the Tammineni et al. method. ${ }^{12}$ For MC, film portions of $2 \times 2 \mathrm{~cm}^{2}$ dimension were weighed and dried in an oven (FED 115, Binder, Germany) at $105{ }^{\circ} \mathrm{C}$ for $24 \mathrm{~h}$. The MC was calculated according to the following equation:

$$
\operatorname{MC}(\%)=\frac{W_{0}-W_{1}}{W_{0}} \times 100 \%
$$

where $W_{0}$ is the initial dry mass of the sample $(\mathrm{g})$ and $W_{1}$ is the final dry mass of the sample (g). All tests were carried out in triplicates.

For FS, samples $(20-30 \mathrm{mg})$ were immersed in a centrifuge tube containing $30 \mathrm{~mL}$ distilled water for $24 \mathrm{~h}$. The tubes were centrifuged for $15 \mathrm{~min}$ at $9000 \mathrm{rpm}$ (centrifuge, Anke TGL-10 C). After this period, the samples were dried in an oven at $105{ }^{\circ} \mathrm{C}$ until a constant weight was achieved. The FS was calculated according to the following equation:

$$
\mathrm{FS}(\%)=\frac{W_{1}-W_{2}}{W_{1}} \times 100 \%
$$

where $W_{1}$ is the initial film weight and $W_{2}$ is the weight of undissolved dry matter.

2.3.3. Water vapor permeability. The water vapor permeability (WVP) was determined by the gravimetric method as described by Sobral et al. ${ }^{13}$ The film was used to cover the mouth of a $50 \mathrm{~mL}$ conical flask containing $20 \mathrm{~mL}$ of distilled water and sealed tightly using a rubber band. This was placed in a desiccator filled with anhydrous silica gel (relative humidity $0 \%$ ) at $22{ }^{\circ} \mathrm{C}$. The exposed area of the film was $9.62 \mathrm{~cm}^{2}$. The samples were weighed at $1 \mathrm{~h}$ intervals over $12 \mathrm{~h}$ periods and the WVP was calculated using the following equation:

$$
\mathrm{WVP}=\frac{\Delta m \times x}{S \times \Delta P \times t}
$$

where $\Delta m$ is the weight gained (mg), $x$ is the average thickness of the film (mm), $t$ is the elapsed time for the weight gained (s), $S$ is the exposed area of the film $\left(\mathrm{m}^{2}\right)$, and $\Delta P$ is the vapor pressure difference across the film ( $\mathrm{Pa})$. All tests were carried out in triplicates.

2.3.4. FT-IR spectroscopy. Fourier-transform infrared (FTIR) spectroscopy analyses were performed in a transmission mode using a spectrometer (NicoletTM iSTM10, Thermo Fisher, USA). The spectral range was $4000-750 \mathrm{~cm}^{-1}$. Each spectrum was collected at a resolution of $4 \mathrm{~cm}^{-1}$.

2.3.5. Structural characterization. A scanning electron microscope (SEM) (JSM 6700F, JEOL, Japan) was used to visualize the surface morphology of film samples. The samples were sputtered with gold for 30 seconds. The photographs were taken at an acceleration voltage of $5 \mathrm{kV}$.

2.3.6. Light transmission. The barrier properties of films against ultraviolet-visible light were performed at selected wavelengths between 200 and $800 \mathrm{~nm}$, using a UV-Vis spectrophotometer (Cary-50, Vivian, Australia).

2.3.7. DPPH free radical-scavenging assay. The film $(200$ $\mathrm{mg}$ ) was mixed with $10 \mathrm{~mL}$ of $95 \%$ ethanol, and then the solution was kept in the dark at $50{ }^{\circ} \mathrm{C}$ for $3 \mathrm{~h} .1 .5 \mathrm{~mL}$ of the supernatant obtained was added to $1.5 \mathrm{~mL}$ of $0.06 \mathrm{mM}$ DPPH in $95 \%$ ethanol solution. After that, the mixture was stirred for $30 \mathrm{~min}$ at room temperature in darkness. The absorbance of the mixture solution was measured at $517 \mathrm{~nm}$ using a spectrophotometer (Cary-60, Agilent, USA). The control film was prepared in the same manner except that $95 \%$ ethanol was used instead of the sample. The test was carried out in triplicate.

2.3.8. Antibacterial activities. The antimicrobial activity of the sample films was assessed against Staphylococcus aureus (S.A.) ATCC 6538 and Candida albicans (B.C.) ATCC 10231 by the agar diffusion method. Microbial strains were inoculated in nutrient broth (NB) at an appropriate temperature for $12 \mathrm{~h}$. Young-type strains $(50 \mu \mathrm{L})$ were coated on solidified Nutrient agar plates. The film was cut into $9 \mathrm{~mm}$ diameter circular discs and placed on the nutrient agar plate's surface. Plates inoculated with Staphylococcus aureus strains were incubated at $37^{\circ} \mathrm{C}$ for $24 \mathrm{~h}$, and Candida albicans was incubated at $28^{\circ} \mathrm{C}$ for $24 \mathrm{~h}$. The antimicrobial activity of the tested microorganisms was evaluated by measuring the antibacterial inhibition zone. Gelatin films without GBE addition was used as the control group. 
2.3.9. Statistical analysis. A statistical test was carried out using the SPSS program (IBM SPSS Statistics 24, USA). One-way analysis of variance (ANOVA) was used for assessing the data. When the value of $p<0.05$, the differences were resolved to be statistically significant.

\section{Results and discussion}

\subsection{Mechanical analysis}

TS and EAB are the main parameters of the mechanical strength of the edible films, which represent the maximum tensile strength and the flexibility of the films, respectively. Table 1 summarizes the TS and EAB data of the control film and GBE gelatin films. The TS of the control film was 23.1 MPa. When 0.5 $\mathrm{g} / 100 \mathrm{~g}$ gelatin of GBE was added, the TS increased to 27.4 MPa. With the increase in the content of GBE, the TS of the composite film reached 38.0 $\mathrm{MPa}$. Wu et al. also reported that the TS of gelatin films increased with the incorporation of green tea extracts into the gelatin film. ${ }^{\mathbf{1 4}}$

The addition of GBE also had effects on the EAB of the films, and the EAB values of the gelatin films were between 10.3 and $14.4 \%$. The mechanical properties of the film were primarily related to the distribution and density of intramolecular and intermolecular interactions, depending on the arrangement of the polymer chains in the network. ${ }^{15}$ Crosslinking between the amino group of the protein and the phenolic group in the extract increases the rigidity of the film, thereby increasing the TS and lowering the EAB of the film. ${ }^{16}$ Similar results were obtained in the research by Hoque in which the polyphenol could affect the $\mathrm{EAB}$ values of the gelatin film incorporated with different plant extracts including cinnamon, clove and star anise. $^{17}$

\subsection{Moisture content and film solubility}

Table 1 also shows the moisture content and film solubility of gelatin films. Both MC and FS of the GBE films decreased with the increase in the amount of GBE compared with that of the control film. The MC of the control film was $13.2 \pm$ $0.53 \%$, which is similar to the result obtained by Ciannamea et $a l .{ }^{18}$ It evidently decreased when GBE was incorporated into a gelatin film. The GBE gelatin films possessed lower MC and FS, which may be due to the hydrophobicity of GBE and its strong molecular structure. This might help to extend the quality assurance period of the film. ${ }^{19}$ Similar results were obtained previously when red cabbage was used as an additive to form gelatin dispersion in film formation. ${ }^{20}$

\subsection{Water vapor permeability}

When the food was exposed to high moisture, it was easily susceptible to spoilage. Therefore, it is required to possess a water barrier towards WVP to be used in food packaging. When the concentration of the GBE was increased to $5.0 \mathrm{~g} /$ $100 \mathrm{~g}$ gelatin, the WVP of the composite films decreased significantly $(p<0.05)$ from $4.3 \pm 0.34 \times 10^{-11} \mathrm{~g} \mathrm{~s}^{-1} \mathrm{~m}^{-1}$ $\mathrm{Pa}^{-1}$ to $3.3 \pm 0.22 \times 10^{-11} \mathrm{~g} \mathrm{~s}^{-1} \mathrm{~m}^{-1} \mathrm{~Pa}^{-1}$ (Table 1). The incorporation of GBE can enhance the hydrophobicity of the films, so the WVP of the films would be limited. ${ }^{21}$ A gelatin film added with GBE was prepared with a lower WVP. Yao et al. also noticed that the WVP decreased in the films based on fish gelatin-chitosan supplemented with D-limonene

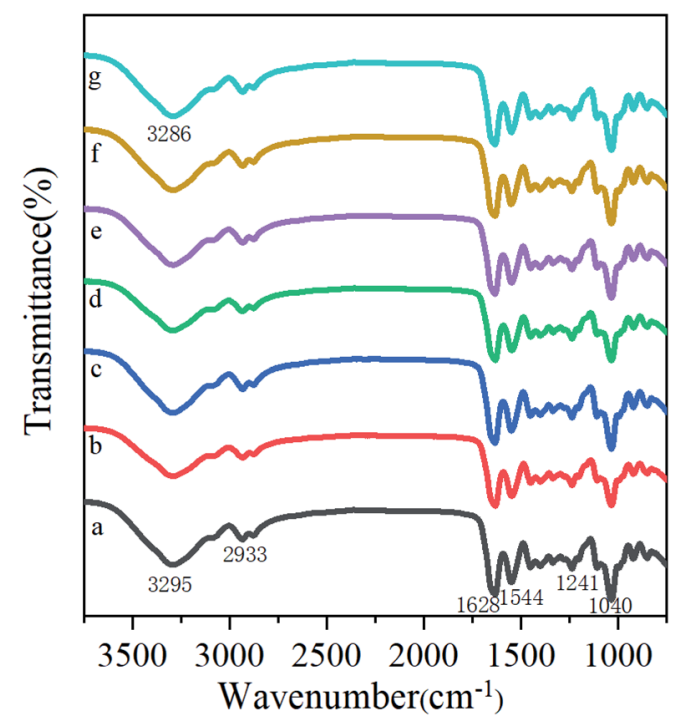

Fig. 1 FTIR spectra of the films. (a) $C_{\mathrm{GBE}}=0 \mathrm{~g}$, (b) $C_{\mathrm{GBE}}=0.5 \mathrm{~g} / 100 \mathrm{~g}$ gelatin, (c) $C_{\mathrm{GBE}}=1.5 \mathrm{~g} / 100 \mathrm{~g}$ gelatin, (d) $C_{\mathrm{GBE}}=2.5 \mathrm{~g} / 100 \mathrm{~g}$ gelatin, (e) $C_{\mathrm{GBE}}=3.0 \mathrm{~g} / 100 \mathrm{~g}$ gelatin, (f) $C_{\mathrm{GBE}}=4.0 \mathrm{~g} / 100 \mathrm{~g}$ gelatin, (g) $C_{\mathrm{GBE}}=5.0$ $\mathrm{g} / 100 \mathrm{~g}$ gelatin.

Table 1 TS, EAB, MC, FS, and WVP of gelatin edible films supplemented with GBE and of the control film ${ }^{a}$

\begin{tabular}{|c|c|c|c|c|c|}
\hline$C_{\mathrm{GBE}}$ & TS (MPa) & $\mathrm{EAB}(\%)$ & MC (\%) & FS (\%) & $\begin{array}{l}\text { WVP }\left(10^{-11} \mathrm{~g}\right. \\
\left.\mathrm{s}^{-1} \mathrm{~m}^{-1} \mathrm{~Pa}^{-1}\right)\end{array}$ \\
\hline 0 & $23.1 \pm 1.46^{\mathrm{e}}$ & $12.8 \pm 1.46^{\mathrm{ab}}$ & $13.2 \pm 0.53^{\mathrm{a}}$ & $36.1 \pm 2.50^{\mathrm{a}}$ & $4.3 \pm 0.34^{\mathrm{a}}$ \\
\hline 0.5 & $27.4 \pm 1.59^{\mathrm{d}}$ & $14.4 \pm 1.59^{\mathrm{a}}$ & $11.9 \pm 0.94^{\mathrm{a}}$ & $34.5 \pm 2.83^{\mathrm{a}}$ & $4.1 \pm 0.15^{\mathrm{b}}$ \\
\hline 1.5 & $31.4 \pm 0.89^{c}$ & $11.4 \pm 0.89^{a b}$ & $10.7 \pm 0.68^{\mathrm{ab}}$ & $33.5 \pm 3.02^{\mathrm{a}}$ & $3.9 \pm 0.41^{\mathrm{c}}$ \\
\hline 2.5 & $33.5 \pm 1.16^{b c}$ & $10.6 \pm 1.16^{b}$ & $10.4 \pm 0.57^{\mathrm{ab}}$ & $31.2 \pm 1.61^{b}$ & $3.7 \pm 0.09^{c}$ \\
\hline 3 & $34.5 \pm 1.27^{\mathrm{abc}}$ & $10.6 \pm 1.27^{\mathrm{b}}$ & $10.0 \pm 0.66^{\mathrm{ab}}$ & $30.3 \pm 1.13^{b}$ & $3.4 \pm 0.10^{\mathrm{d}}$ \\
\hline 4 & $36.3 \pm 1.41^{\mathrm{ab}}$ & $11.5 \pm 1.41^{\mathrm{ab}}$ & $9.4 \pm 0.22^{\mathrm{b}}$ & $29.9 \pm 1.02^{c}$ & $3.4 \pm 0.02^{\mathrm{d}}$ \\
\hline 5 & $38.0 \pm 1.53^{\mathrm{a}}$ & $10.3 \pm 1.53^{\mathrm{b}}$ & $8.8 \pm 0.92^{b}$ & $28.1 \pm 0.19^{c}$ & $3.3 \pm 0.22^{\mathrm{d}}$ \\
\hline
\end{tabular}

${ }^{a}$ The superscripted letters represent SPSS single factor analysis. 
possibly because D-limonene was in a hydrophobic oil phase, and hence, it can decrease the water transfer through films. ${ }^{19}$

\subsection{FT-IR analysis}

he infrared spectra of the gelatin films containing GBE at different concentrations are exhibited in Fig. 1. The major peaks of the control film (Fig. 1a) are present at $3295 \mathrm{~cm}^{-1}$ (amide-A, N-H stretching), $2933 \mathrm{~cm}^{-1}$ (amide-B, C-H stretching), $1628 \mathrm{~cm}^{-1}$ (amide-I, $\mathrm{C}=\mathrm{O}$ stretching), $1544 \mathrm{~cm}^{-1}$ (amide-II, N-H bending), and $1241 \mathrm{~cm}^{-1}$ (amideIII, $\mathrm{C}-\mathrm{N}$ and $\mathrm{N}-\mathrm{H}$ stretching). The addition of GBE to the gelatin film also displayed characteristic peaks in the spectral region, which was similar to that of the control film. As can be seen in Fig. 1, the spectrum of the films incorporated with different concentrations of GBE showed that some of the peaks shifted to lower or higher wavenumbers in comparison with the spectrum of the control film. The amide A peak shifted to lower wavenumbers, which could be attributed to free $\mathrm{O}-\mathrm{H}$ and $\mathrm{N}-\mathrm{H}$ groups forming hydrogen bonding with the carbonyl group in the films. Moreover, amide-I could be observed at lower wavenumbers, presumably due to the disordered molecular structure. A band was found at the wavenumber of $1040 \mathrm{~cm}^{-1}$ in the spectra of all films. It was possibly associated with a skeletal stretching vibration of $\mathrm{C}=\mathrm{C}$ bonds. Therefore, the results illustrated that the addition of GBE might alter the intermolecular interactions in the gelatin films.

\subsection{Film microstructure}

To further understand the internal interactions of the gelatin film, the SEM microstructures of the gelatin edible films were obtained and are displayed in Fig. 2. The control film showed a compact, smooth and homogeneous surface, which was comparable to the previous study. ${ }^{22}$ After adding GBE, the surface of the gelatin films presented similar characteristics. However, with a higher concentration of GBE added, more pores and cavities appeared in the films. It was evident that there are some aggregates of GBE at the highest concentration of GBE added. As a result, a homogeneous dispersion of GBE
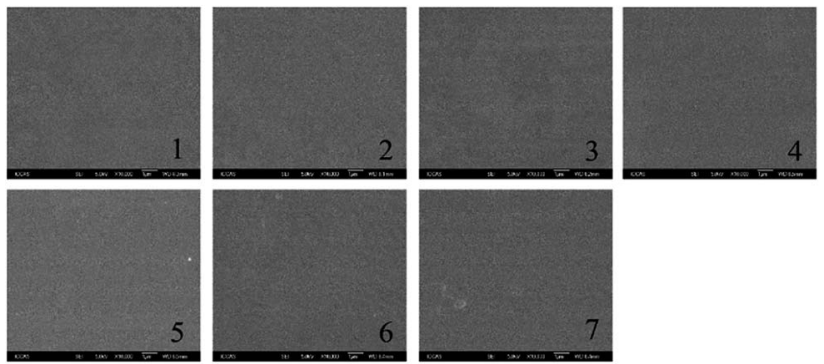

Fig. 2 SEM micrographs of the surface of the films. (1) $C_{\mathrm{GBE}}=0 \mathrm{~g}$, (2) $C_{\mathrm{GBE}}=0.5 \mathrm{~g} / 100 \mathrm{~g}$ gelatin, (3) $C_{\mathrm{GBE}}=1.5 \mathrm{~g} / 100 \mathrm{~g}$ gelatin, (4) $C_{\mathrm{GBE}}=2.5$ $\mathrm{g} / 100 \mathrm{~g}$ gelatin, (5) $C_{\mathrm{GBE}}=3.0 \mathrm{~g} / 100 \mathrm{~g}$ gelatin, (6) $C_{\mathrm{GBE}}=4.0 \mathrm{~g} / 100 \mathrm{~g}$ gelatin, (7) $C_{\mathrm{GBE}}=5.0 \mathrm{~g} / 100 \mathrm{~g}$ gelatin. would show a better mechanical property. Otherwise, it would induce phase separation and affect some properties. Hanani et al. also reported that the control film prepared by gelatin exhibited more compact internal structures than the films with pomegranate peel powder. ${ }^{23}$

\subsection{Ultraviolet and visible light barrier properties}

Food oxidation is the main cause of food corruption in production and storage. Nowadays, it has attracted wide attention by adding anti-ultraviolet additives to food packaging films. Bitencourt et al. reported that food packaging with high UV/visible barrier can prevent the lipid oxidation of food. ${ }^{24}$ Light transmission from 200 to $800 \mathrm{~nm}$ in UV and visible ranges of films from gelatin containing glycerol (20\%) and various concentrations of GBE are shown in Fig. 3. Regardless of the concentrations of GBE, the transmittance of UV light was very low at 200 and $240 \mathrm{~nm}$ for all films, indicating that the GBE gelatin films possess high barrier to UV. This may be related to the chemical structure of GBE. In addition to the aromatic amino acids in gelatin molecules, GBE also exhibits phenolic absorption in the ultraviolet range. Compared with the control film, the addition of GBE reduces the transmittance of the film in the UV-visible region (Fig. 3). In other words, the addition of GBE increases the UV-visible shielding performance of the film. The reduction in light transmission can be attributed to flavonoids and ginkgolides present in the GBE. There are numerous benzene rings, $-\mathrm{OH}$ containing polyphenol compounds, $\mathrm{C}=\mathrm{O}$ in flavonoids and ginkgo lactone, which improve the $\mathrm{n} \rightarrow \pi^{*}$ absorption in the ultraviolet region. This result is similar to the investigation of Dammak et al. They reported that gelatin films incorporated with rutin-loaded nanoemulsions had good barrier property towards the light in the UV region at 200$280 \mathrm{~nm}^{.22}$

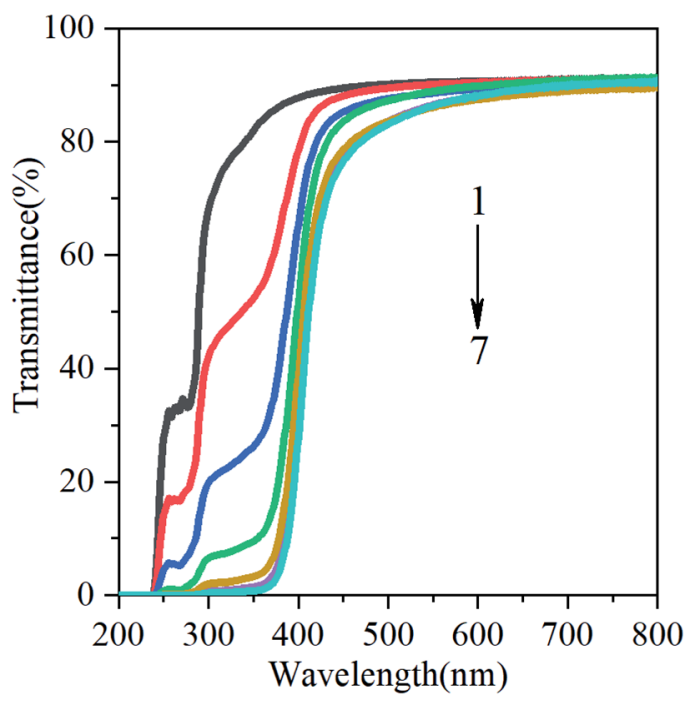

Fig. 3 Ultraviolet and visible light barrier properties of films. (1) $C_{\mathrm{GBE}}=$ $0 \mathrm{~g}$, (2) $C_{\mathrm{GBE}}=0.5 \mathrm{~g} / 100 \mathrm{~g}$ gelatin, (3) $C_{\mathrm{GBE}}=1.5 \mathrm{~g} / 100 \mathrm{~g}$ gelatin, (4) $C_{\mathrm{GBE}}=2.5 \mathrm{~g} / 100 \mathrm{~g}$ gelatin, (5) $C_{\mathrm{GBE}}=3.0 \mathrm{~g} / 100 \mathrm{~g}$ gelatin, (6) $C_{\mathrm{GBE}}=$ $4.0 \mathrm{~g} / 100 \mathrm{~g}$ gelatin, (7) $C_{\mathrm{GBE}}=5.0 \mathrm{~g} / 100 \mathrm{~g}$ gelatin. 


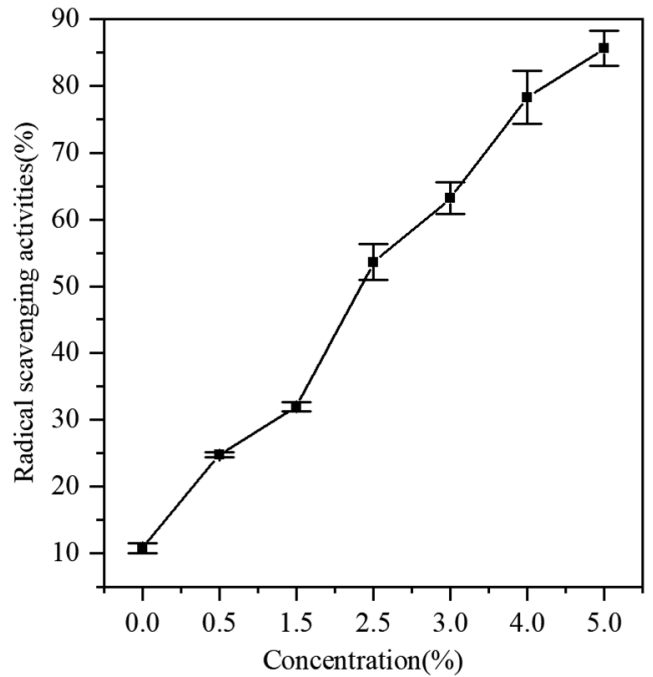

Fig. 4 DPPH radical scavenging activity of films.

\subsection{Antioxidant activity}

At present, it is generally considered that an important method to determine the antioxidant activity is the scavenging ability of DPPH free radicals. Fig. 4 shows the antioxidant activity of gelatin films with different concentrations of GBE. The films incorporated with GBE showed higher antioxidant activities than the control film. GBE has been reported as a great source of antioxidants. ${ }^{25}$ Adding antioxidants to active packaging is a promising technique for prolonging the shelf life of food. Furthermore, antioxidants can improve nutritional and aesthetic qualities without affecting food integrity. Compared with the control film, GBE films showed that the radical scavenging activity was $24.7 \%\left(C_{\mathrm{GBE}}=0.5 \mathrm{~g} / 100 \mathrm{~g}\right.$ gelatin $)$. With the
Table 2 Antibacterial activity of edible films with different concentrations of GBE and of the control film

\begin{tabular}{lll}
\hline$C_{\mathrm{GBE}}$ & $\begin{array}{l}\text { Inhibition zone } \\
\text { for S.A. }(\mathrm{mm})\end{array}$ & $\begin{array}{l}\text { Inhibition zone } \\
\text { for B.C. }(\mathrm{mm})\end{array}$ \\
\hline 0 & Not detected & Not detected \\
0.5 & $10.8 \pm 0.08^{\mathrm{c}}$ & $\begin{array}{l}\text { Not detected } \\
1.5\end{array}$ \\
2.5 & $11.3 \pm 0.11^{\mathrm{c}}$ & Not detected \\
3 & $12.6 \pm 0.10^{\mathrm{b}}$ & $13.5 \pm 0.22^{\mathrm{b}}$ \\
4 & $13.1 \pm 0.05^{\mathrm{a}}$ & $13.8 \pm 0.16^{\mathrm{b}}$ \\
5 & $13.2 \pm 0.04^{\mathrm{a}}$ & $19.2 \pm 0.46^{\mathrm{a}}$ \\
& $13.6 \pm 0.34^{\mathrm{a}}$ & $20.3 \pm 0.20^{\mathrm{a}}$
\end{tabular}

increase in the concentration of GBE, the free radical scavenging effect ranged from $24.7 \%$ to $85.6 \%$. The results indicated that the gelatin film containing GBE could be used as the active film with antioxidant activity.

\subsection{Antibacterial activity}

The antibacterial activities of gelatin films containing GBE against Staphylococcus aureus and Candida albicans bacteria are displayed in Fig. 5. Table 2 lists the zone of inhibition. The results presented that the control film did not inhibit the growth of the test microorganisms. As the concentration of GBE increased in the gelatin film, the zone of inhibition enlarged significantly $(p<0.05)$. Table 2 shows that the zone of inhibition significantly increased from a non-detectable area and $10.8 \mathrm{~mm}$ (0.5 g GBE/100 g gelatin) to 20.3 and $13.6 \mathrm{~mm} \mathrm{(5} \mathrm{g} \mathrm{GBE/100} \mathrm{g}$ gelatin) against Candida albicans and Staphylococcus aureus, respectively. Phenolic compounds could destroy the integrity of bacterial walls and cell membranes, leading to the release of intracellular components in microbial cells. It would cause some functional obstacles such as electron transfer, nutrient
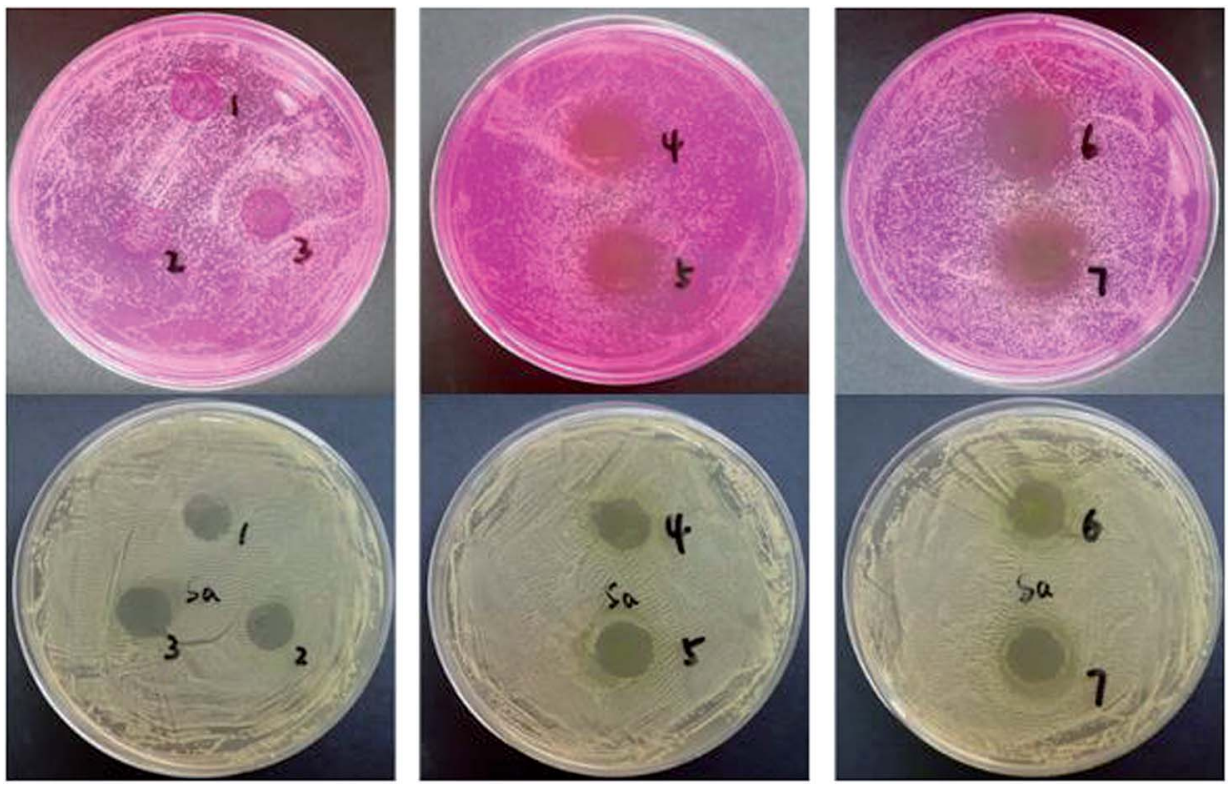

Fig. 5 Photographs of the antibacterial activity on two tested strains. (1) $C_{\mathrm{GBE}}=0 \mathrm{~g}$, (2) $C_{\mathrm{GBE}}=0.5 \mathrm{~g} / 100 \mathrm{~g}$ gelatin, (3) $C_{\mathrm{GBE}}=1.5 \mathrm{~g} / 100 \mathrm{~g}$ gelatin, (4) $C_{\mathrm{GBE}}=2.5 \mathrm{~g} / 100 \mathrm{~g}$ gelatin, (5) $C_{\mathrm{GBE}}=3.0 \mathrm{~g} / 100 \mathrm{~g}$ gelatin, (6) $C_{\mathrm{GBE}}=4.0 \mathrm{~g} / 100 \mathrm{~g}$ gelatin, (7) $C_{\mathrm{GBE}}=5.0 \mathrm{~g} / 100 \mathrm{~g}$ gelatin. 
absorption, nucleotide synthesis and ATP activity in membranes. Therefore, the growth of microorganisms was inhibited. However, the antimicrobial mechanism of GBE should be further explored. Kavoosi et al. noted that the film added with carvacrol had great antibacterial properties. As essential oils possess the ability to destroy the lipid structure of bacterial cell walls, the cell membranes break first, followed by cytoplasmic leakage, cell lysis, and ultimately cell death. ${ }^{26}$ Liu et al. found that $\mathrm{ZnO}$ nanoparticles added to gelatin/chitosan films endows the films with strong antimicrobial activities. The bacteriostatic mechanism was due to the destruction of microbial cells by the highly active free radicals of zinc oxide. ${ }^{27}$ Overall, the investigation showed that the gelatin films incorporated with GBE had potential application against target microorganisms.

\section{Conclusions}

Edible films with antibacterial and antioxidant activities have been fabricated by incorporating GBE into gelatin. The GBE in the films can interact with the gelatin in the matrix. The interaction affected the mechanical properties, microstructure and barrier properties of the films. Incorporation of the GBE into gelatin films decreased the moisture content and improved the thermal stability and antimicrobial properties, which was a great prospect for its application in food packaging. The antioxidant activities monitored by the DPPH method showed high activities after the incorporation of GBE. Packaging films made from GBE and gelatin provide a new way to inhibit microbial activities, delay lipid oxidation and prolong the shelflife of foods.

\section{Conflicts of interest}

There are no conflicts to declare.

\section{References}

1 E. Dorta, M. G. Lobo and M. González, LWT-Food Sci. Technol., 2012, 45, 261-268.

2 K. De Clercq, C. Schelfhout, M. Bracke, O. De Wever, M. Van Bockstal, W. Ceelen, J. P. Remon and C. Vervaet, Biomaterials, 2016, 96, 33-46.

3 A. Valdes, A. C. Mellinas, M. Ramos, N. Burgos, A. Jimenez and M. C. Garrigos, RSC Adv., 2015, 5, 40324-40335.

4 S. Shankar, L. Jaiswal, P. R. Selvakannan, K. S. Ham and J. W. Rhim, RSC Adv., 2016, 6, 67340-67352.

5 S. H. Yu, H. Y. Hsieh, J. C. Pang, D. W. Tang, C. M. Shih, M. L. Tsai, Y. C. Tsai and F. L. Mi, Food Hydrocolloids, 2013, 32, 9-19.
6 M. Jouki, S. A. Mortazavi, F. T. Yazdi and A. Koocheki, Carbohydr. Polym., 2014, 99, 537-546.

7 W. Gong, C. Chen, C. Dobes, C. X. Fu and M. A. Koch, Mol. Phylogenet. Evol., 2008, 48, 1094-1105.

8 B. Ahlemeyer and J. Krieglstein, ACS Symp. Ser., 2003, 60, 1779-1792.

9 P. Sati, P. Dhyani, I. D. Bhatt and A. Pandey, J. Tradit. Complement. Med., 2019, 9, 15-23.

10 J. H. Li, J. Miao, J. L. Wu, S. F. Chen and Q. Q. Zhang, Food Hydrocolloids, 2014, 37, 166-173.

11 K. I. Iwata, S. H. Ishizaki, A. K. Handa and M. U. Tanaka, Fish. Sci., 2000, 66, 372-378.

12 N. Tammineni, G. Unlu, B. Rasco, J. Powers, S. Sablani and C. Nindo, J. Food Sci., 2012, 77, 342-347.

13 P. J. A. Sobral, F. C. Menegalli, M. D. Hubinger and M. A. Roques, Food Hydrocolloids, 2001, 15, 423-432.

14 J. L. Wu, S. F. Chen, S. Y. Ge, J. Miao, J. H. Li and Q. Q. Zhang, Food Hydrocolloids, 2013, 32, 42-51.

15 M. Ahmad, N. M. Hani, N. P. Nirmal, F. F. Fazial, N. F. Mohtar and S. R. Romli, Prog. Org. Coat., 2015, 84, 115-127.

16 Z. A. Maryam Adilah, B. Jamilah and Z. A. Nur Hanani, Food Hydrocolloids, 2018, 74, 207-218.

17 M. S. Hoque, S. Benjakul and T. Prodpran, Food Hydrocolloids, 2011, 25, 1085-1097.

18 E. M. Ciannamea, P. M. Stefani and R. A. Ruseckaite, $L W T-$ Food Sci. Technol., 2016, 74, 353-362.

19 Y. Yao, D. Ding, H. Shao, Q. Peng and Y. Huang, Int. J. Polym. Sci., 2017, 2017, 1-9.

20 Y. S. Musso, P. R. Salgado and A. N. Mauri, Food Hydrocolloids, 2019, 89, 674-681.

21 Z. A. Maryam Adilah, B. Jamilah and Z. A. Nur Hanani, Food Hydrocolloids, 2018, 74, 207-218.

22 I. Dammak, R. A. de Carvalho, C. S. Trindade, R. V. Lourenço and P. J. de Amaral Sobral, Int. J. Biol. Macromol., 2017, 98, 39-49.

23 Z. A. N. Hanani, F. C. Yee and M. A. R. Nor-Khaizura, Food Hydrocolloids, 2019, 89, 253-259.

24 C. M. Bitencourt, C. S. Fávaro-Trindade, P. J. A. Sobral and R. A. Carvalho, Food Hydrocolloids, 2014, 40, 145-152.

25 J. Y. Han, D. M. Xing, S. Hong, L. U. Hong, L. I. Min and D. U. Li-Jun, Chin. Pharmacol. Bull., 2002, 18, 115-117.

26 G. Kavoosi, S. M. M. Dadfar, A. M. Purfard and R. Mehrabi, J. Food Saf., 2013, 33, 423-432.

27 Z. Liu, M. Lv, F. Li and M. Zeng, J. Aquat. Food Prod. Technol., 2016, 25, 1056-1063. 